\title{
Compliance to oral nutritional supplements and its association with increased total energy intake: results from a systematic review
}

\author{
G. P. Hubbard ${ }^{1}$, M. Elia ${ }^{2}$, A. Holdoway ${ }^{3}$ and R. J. Stratton ${ }^{1}$ \\ ${ }^{1}$ Medical Affairs, Nutricia, Trowbridge, Wiltshire, BA14 OXQ,${ }^{2}$ Institute of Human Nutrition, University of Southampton, \\ Southampton, SO16 6YD and ${ }^{3}$ Nutrition \& Dietetic Services, Great Western Hospitals NHS Foundation Trust and Wiltshire \\ Community Health Services, Marlborough Road, SN3 6BB, UK
}

\begin{abstract}
As there is uncertainty about compliance with oral nutritional supplements (ONS) and the inter-relationship with ONS intake, energy intake from food and total energy intake, this was investigated as part of a systematic review on compliance to ONS.

This systematic review using searches of electronic databases and bibliographies (up to October 2009) ${ }^{(1)}$ identified 46 eligible studies (n 4328) of multi-nutrient ONS (containing macronutrients plus micronutrients) where mean \% compliance (ONS consumed vs. prescribed) was reported or could be calculated. Energy intake from food (EIF) and total energy intake (energy intake from food plus ONS energy intake (TEI)) were recorded. The percentage of ONS energy intake that was additive to food intake was calculated using published methods ${ }^{(2)}$. Thirty three studies involved patients in community settings (n 2604), with the remaining studies being undertaken in hospitals (10 studies, $\mathrm{n}$ 1497) or multiple settings (3 studies, n 227). Thirty two studies were randomised controlled trials, with the remaining 14 being non-randomised trials, of which 5 were surveys of ONS usage in clinical practice. Study control groups included routine care (12 studies), mixed interventions (9 studies), another ONS (7 studies), placebo (5 studies) or dietary advice (4 studies) and 9 studies had no control group.

Mean ONS compliance was 78\% (SD 15) (37\%-100\%; $81 \%$ in community; $67 \%$ in hospitals). Percentage compliance was similar in randomised $(79 \%)$ and non-randomised $(77 \%)$ trials (61\% in surveys). Compliance to ONS across a range of heterogeneous studies was positively correlated with ONS energy intake (mean $433 \mathrm{kcal} / \mathrm{d}(\mathrm{SD} 196))\left(r^{2}=0.106, p=0.024\right)$ and TEI at the end of studies $\left(r^{2}=0.307\right.$, $p=0.002)$. Consumption of ONS significantly increased TEI (1898 kcal/d (SD 365)) compared to baseline EIF (1455 kcal/d (SD 219) $p=0.001,10$ studies) and end of study EIF (1393 kcal/d (SD 172) $p=0.0001,10$ studies). Similar improvements in TEI were not seen in the unsupplemented control groups (1604 kcal/d (SD 302)) vs. baseline EIF (1531 kcal/d (SD 418)) $p=0.239,9$ studies). Meta-analysis of 8 RCTs ( $n$ 771) indicated significantly greater total energy intakes in the ONS group compared to the unsupplemented control groups (difference in means: $375 \mathrm{kcal} / \mathrm{d}$ (SEM 72), random effects model, $p=0.0001$ ). Mean percentage of ONS energy intake that was additive to food was $102 \%$ (SD 43, range 31-209\%, in 13 studies). Consumption of ONS did not effect EIF which was not significantly different between baseline (1455 kcal/d (SD 219)) and end of study period (1393 kcal/d (SD 172)) $p=0.455,10$ studies). A borderline significant positive correlation between compliance and EIF alone at the end of studies was also observed $\left(r^{2}=0.182, p=0.069\right.$, 19 studies).

This systematic review suggests that: (i) Compliance to ONS is good (78\%) under the conditions of a wide range of studies; (ii) Compliance to ONS in heterogeneous groups of patients is positively associated with ONS energy intake and increased total energy intake; (iii) ONS in general appear to have little if any effect in suppressing voluntary food intake.
\end{abstract}

1. The Cochrane Collaboration. Cochrane Reviewers Handbook 4.2.5 (handbook online). 2005.

2. Stratton RJ, Green CJ \& Elia M (2003) Disease-related malnutrition: an evidence-based approach to treatment. Oxford: CABI publishing. 